\title{
Selectivity Improvement of Gas Sensor Based on Poly(3,4-ethylenedioxythiophene):Poly(styrenesulfonate) Thin Film by Using Imprinting Method
}

\author{
La Aba $^{1,2}$, Yusril Yusuf ${ }^{1}$, Mitrayana ${ }^{1}$, Kuwat Triyana ${ }^{1}$ \\ ${ }^{1}$ Physics Department, Gadjah Mada University, Yogyakarta, Indonesia \\ ${ }^{2}$ Physics Department, Haluoleo University, Kendari, Indonesia \\ Email: la.aba@mail.ugm.ac.id, laabaz@yahoo.com
}

Received March 28, 2012; revised April 30, 2012; accepted May 16, 2012

\begin{abstract}
Selectivity improvement of gas sensor based on Poly(3,4-ethylenedioxythiophene):poly(styrenesulfonate) (PEDOT:PSS) thin film to ammonia gas has been studied. The PEDOT:PSS thin films were deposited on glass and FR4 substrates by using a spin-coating technique. PEDOT:PSS solution was spread on the substrate followed by spinning at fixed rate. Then, the imprinting process of gas sensor was performed by injecting ammonia gas into the spin-coating chamber. Finally, the gas sensor was dried on a controllable hot plate. Current-voltage characteristics of the films were measured by using calibrated electrometer. The results show that by imprinting of ammonia gas to the film affects the electrical conductivity of the film. It is also shown that the electron transport in the PEDOT:PSS thin film tends to be ohmic-contact. When the imprinted sensor is exposed in ammonia gas, we obtained that the sensor has short response and recovery time, a good repeatability (reversible), and higher sensitivity to ammonia gas. To this end, we found that ammonia imprinting on the surface of PEDOT:PSS thin film can improve the selectivity of the sensor to ammonia gas. It indicates that our method can be used for fabricating the sensor which has a single selectivity.
\end{abstract}

Keywords: PEDOT:PSS Thin Film; Imprinting; Ammonia Gas; Sensitivity; Selectivity

\section{Introduction}

The gas sensors have been extensively used for qualitative and quantitative detections of chemical substances especially for environmental monitoring, personal protection, safety and process control [1-3]. For this purpose, many attempts have done in order to fabricate the suitable sensing materials. A wide range of materials both from organic and inorganic substances are usually used as an active layer of gas sensing elements. The inorganic materials based of metal-oxide such as oxides of tin and zinc have been used extensively [4]. However, one of practical problems with oxide gas sensors is that it needs a high operating temperatures in the range of $250^{\circ} \mathrm{C}$ $300^{\circ} \mathrm{C}$. This might be substituted by using the organic materials, because sensor based on the organic compounds can operate at ambient temperature. For this purpose, several types of organic materials that is porphyrins and phthalocyanines have been used [5]. The conducting polymers such as polypyrrole (PPy), polyaniline (Pani), polythiophene (PTh) and their derivatives are also possible [6,7]. The gas sensors made from conducting polymers have several advantageous. For example, it has high sensitivity and short response time at room temperature.
Moreover, conducting polymers are easy to synthesize through chemical or electrochemical processes. Their molecular chain structure can also be modified conveniently by copolymerization or structural derivations. In addition, conducting polymers have also good mechaniccal properties, which allow a facile fabrication of gas sensors [7].

In the present study, we used a conducting polymer of Poly(3,4-ethylenedioxythiophene) doped with poly(styrenesulfonate) (PEDOT:PSS). The PEDOT:PSS has similar characteristics with other conducting polymers like polyaniline and polypirrole. It also possesses several advantageous properties such as it has a low oxidation potential, high conductivity and environmental stability [8]. Unfortunately, the recent gas sensors based on PEDOT: PSS of Dan et al. [9] have a global selectivity, where it has the responses not only to ammonia gas but also to other gases such as nitrogen dioxide, acetone, methanol, ethanol, and other organic toxic volatile compounds. Sensor with global selectivity is only appropriate for olfactory systems or electronic noses. Because for such electronic nose application, the sensor arrays must produce a unique pattern which represents a specific sub- 
stance. But it is not appropriate for single gas sensor application.

In this paper, we report our attempts to enhance the selectivity of PEDOT:PSS based gas sensor to ammonia gas. For this purpose, we introduce an imprinting method during preparation process of PEDOT:PSS thin film. This method is a technical procedure to form cavities on surface of polymer matrix [10].

\section{Experimental Method}

The conducting polymer used in this study was PEDOT: PSS purchased from H.C. Starck of Clevios PH500. The molecular structure of such PEDOT:PSS is shown in Figure 1.

Before depositing PEDOT:PSS thin films to the FR4 substrates coated with patterned gold (Au) electrodes (Figure 2(a)), the substrates were cleaned by using a standard procedure of Schweizer [8]. The PEDOT:PSS thin films were then deposited on the substrates by using a spin-coating technique. PEDOT:PSS solution was spread

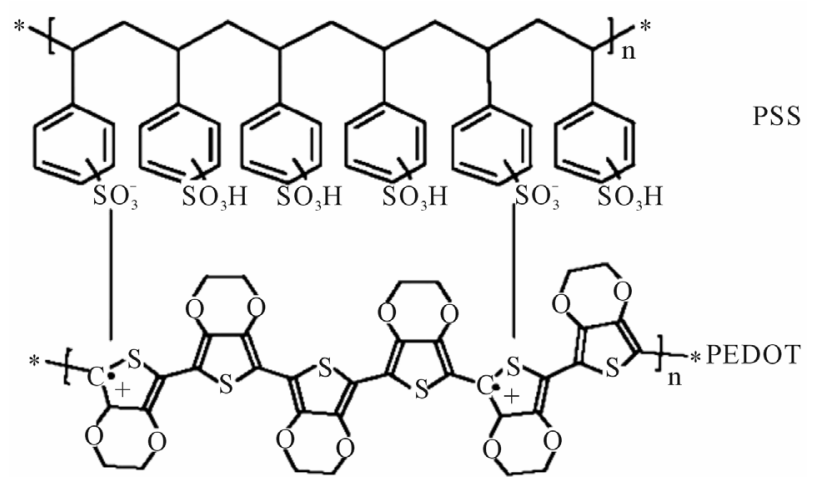

Figure 1. Molecular structure of PEDOT and PSS, respectively.

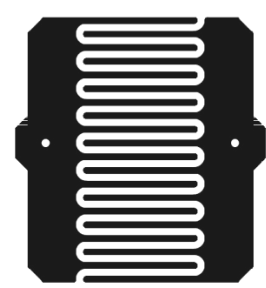

(a)

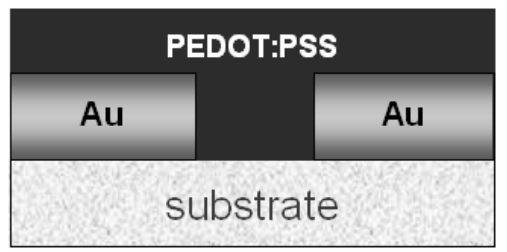

(b)

Figure 2. Schematic of (a) Top view of FR4 substrate with Au patterned electrode; (b) Cross section of the gas sensor. on the substrate followed by spinning at $1000 \mathrm{rpm}$ for 20 seconds. Then, the imprinting process of gas sensor was performed by injecting ammonia gas (about 400 ppm) into the spin-coating chamber for about 5 minutes. Finally, the gas sensor was dried on a controllable hot plate at $60^{\circ} \mathrm{C}$ for about 5 minutes. The cross section of gas sensor is shown in Figure 2(b).

Furthermore, the fabricated gas sensors were annealed at $100^{\circ} \mathrm{C}$ for 30 minutes to vaporize the solvent and ammonia that was imprinted in the films. The fabricated devices were placed in a controllable dry box. The current-voltage $(\mathrm{I}-\mathrm{V})$ characteristics of the films were then measured by using calibrated electrometer [11,12]. We then measure the electrical resistance of the sensor in ammonia $(R)$ and in the ambient air $\left(R_{0}\right)$ in order to understand the effect of the imprinting in connection with the respond to the ammonia gas.

\section{Results and Discussion}

First we discuss the I-V characteristic of the PEDOT:PSS thin films. Figure 3 shows the obtained I-V characteristic of the thin film. We can see that the flowing current through the film is increase linearly with the increasing of the voltage of the electrodes. It means that the contact between PEDOT:PSS layer and Au electrode is an ohmic contact [13].

The effect of imprinting on conductivity of PEDOT: PSS based gas sensors is given in Figure 3. It is found that the increase of ammonia concentration during imprinting process increase the conductivity of gas sensors. This phenomenon might be explained as follow. During imprinting process the ammonia molecules are adsorbed by PEDOT:PSS thin film based on diffusion mechanism. It causes transfer and exchange charges between ammonia molecules and the PEDOT:PSS films. The similar explanation of this mechanism has also been described

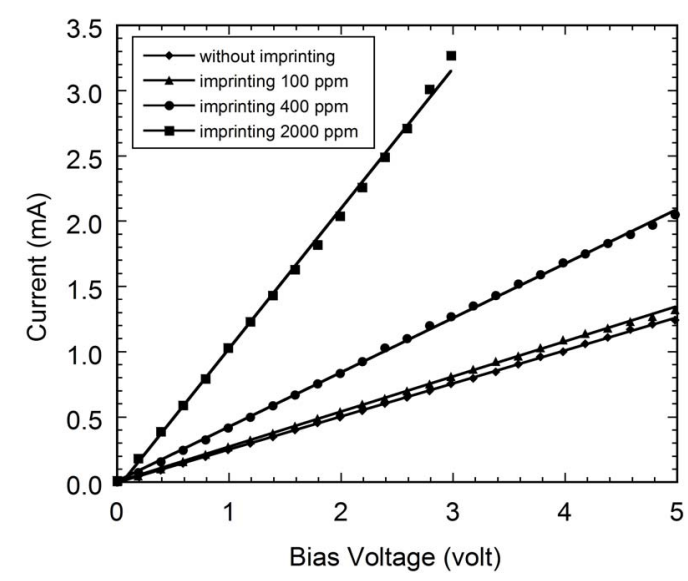

Figure 3. Current-voltage characteristics of the PEDOT: PSS thin films based gas sensor with and without ammonia imprinting (100 ppm, 400 ppm, and 2000 ppm). 
elsewhere [14]. Mechanism of sensing for such conducting polymer devices has been also proposed previously [15] where this mechanism includes the redox reactions between the analyte and the polymer, partially charge transfer between the polymer and the analyte, and polymer swelling.

In this study, due to the chemical nature of ammonia gas, the increasing of sensor resistance could be attributed due to the redox reaction or the charge transfer. Ammonia gas is known to be highly active and electron donating free radical. Therefore, upon electron transfer from ammonia to the partially positive charged sulfur sites on the backbone of the p-type PEDOT:PSS, the charge carrier concentration on the polymer backbone decreases, yielding the increase in the resistance of the PEDOT:PSS films. However, when samples were annealed then ammonia molecules in PEDOT:PSS matrix would evaporate and left both cavities on surface of PEDOT:PSS thin film and holes along the backbone of the polymer. The increase charge carrier concentration in PEDOT:PSS, results in decreasing the resistance. The more vaporized ammonia molecules, the higher conductivity of PEDOT:PSS thin film.

Response of the sensor was defined by normalized resistance, $\left(R-R_{0}\right) / R$, where $R$ is electrical resistance in ammonia gas and $R_{0}$ is resistance in ambient air. Figure 4 shows response of sensors fabricated with and without ammonia imprinting which is exposed in saturated ammonia gas. It can be seen that the response of sensor with ammonia imprinting is higher than the one without ammonia imprinting which indicated that the sensor is more sensitive. This can be the case because both of cavities and holes in PEDOT:PSS thin film adsorb the ammonia gas that contributes for increasing the resistance of sensor.

Figure 5 shows the response of imprinted sensor which is exposed in different concentration of ammonia

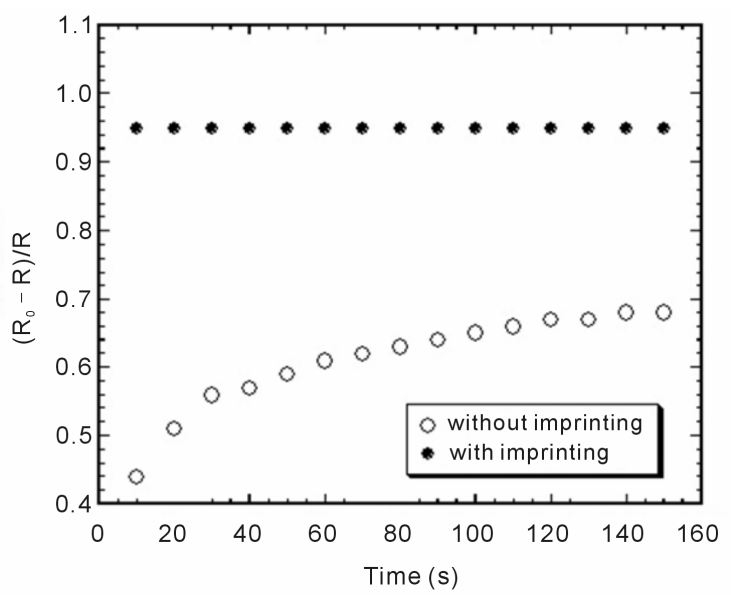

Figure 4. Response of sensors without and with imprinting of 400 ppm ammonia which exposed in ammonia gas. gas. We found that the response of the sensor is increase with the increasing of the concentration of the ammonia gas. In order to see the tendency of this increasing, we plot the correlation of sensor response versus the concentration of the gas. The response of the sensor for the certain concentration is taken as average value in the plateau line in Figure 5. It is shown in Figure 6. We can see that the response of the sensor increases linearly. It is well known that the sensitivity of the sensor is correlated with the slope of the graph shown in the figure. And we obtained the sensitivity of sensor is about $0.02 \% / \mathrm{ppm}$.

The repeatability of the sensor can be understood by measuring the resistance of imprinted sensor as a function of measurement time. The result is shown in Figure 7. The cycles characteristic indicate that the sensor rapidly responds and releases ammonia gas. It means that the sensor has a good repeatability to ammonia gas. This is indicate that the PEDOT:PSS thin film is prominent to be used as an active material of the sensing ammonia gas. We can also see from Figure 7 that the resistance of the sensor increases drastically after being exposed with ammonia gas (in). It indicates that the PEDOT:PSS thin film of sensor is dedoped when detecting ammonia gas. It is because ammonia is an electron-donor type [7]. On

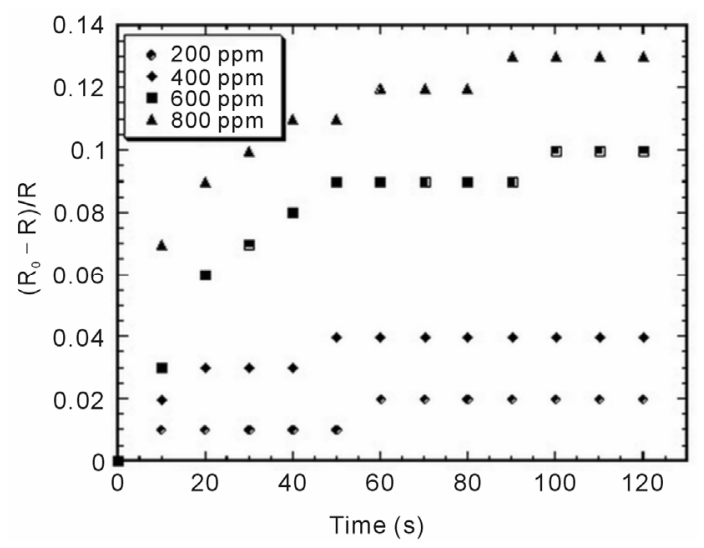

Figure 5. Response of imprinted sensors which was exposed in different concentration of ammonia gas.

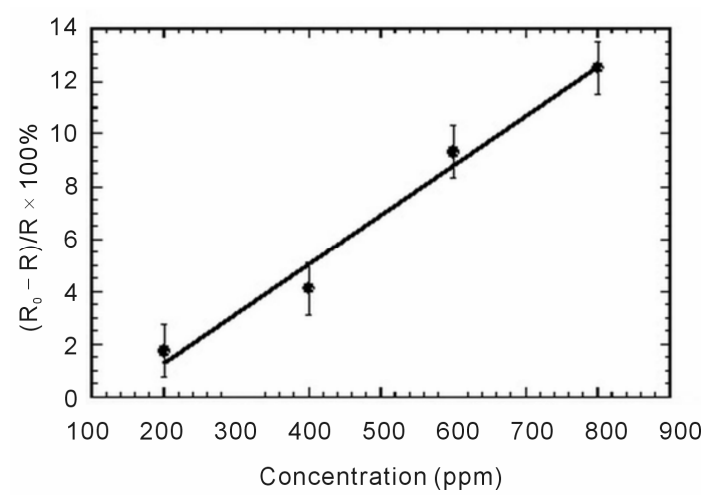

Figure 6. Sensitivity of imprinted sensor. 


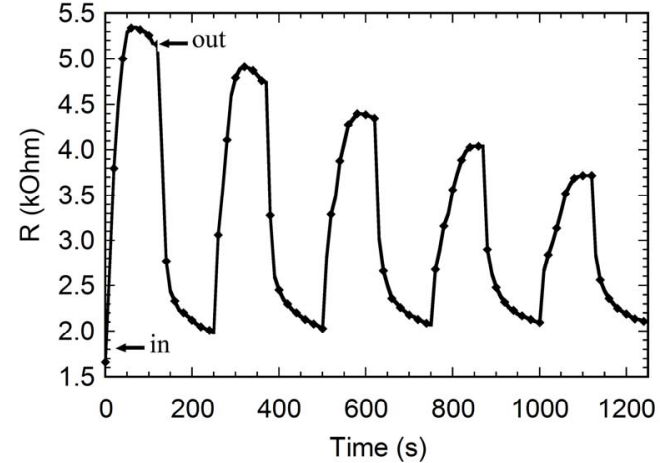

Figure 7. Time dependence of resistance, $R$ of PEDOT:PSS thin film on repeated exposure and removal of ammonia gas.

the other hand, the resistance decreases quickly when ammonia gas is pulled out from the chamber (out). This fact shows that the sensor response is reversible. Furthermore, the decreasing of the response may be caused by decreasing concentration of ammonia gas flowing through the chamber. We also explore the response time and the recovery time of the sensor. The response time was measured under exposure ammonia gas in chamber. And the recovery time was measured when the ammonia gas is pulled out from the chamber by using vacuum pump. We define the response time as a time required to reach $90 \%$ of the saturation value of $R$, while the recovery time as a time needed for $R$ to return to $R_{o}$ on removal of ammonia gas. From Figure 7, we obtained that the response time is about 10 seconds, whereas the recovery time is about 15 seconds.

Finally, we investigated the response of sensor based on PEDOT:PSS thin film with and without ammonia imprinting to several gases, i.e. acetone, methanol, and ammonia in order to see the selectivity of the sensor. The result is given in Figure 8. The figure shows that both sensors (with and without ammonia imprinting) have good selectivity to ammonia gas rather than the others. However, the imprinting treatment also improves the response to ammonia gas and reduces the response to the other gases. This can be the case because the imprinted sensor has the cavities which only appropriate for responding the ammonia gas. These results indicated that ammonia imprinting on the surface of PEDOT:PSS thin film can improve the sensitivity and the selectivity of the sensor to ammonia gas.

\section{Conclusion}

We have fabricated gas sensors based on PEDOT:PSS thin films with and without ammonia imprinting by using spin-coating technique. As results, imprinting of ammonia gas to the film affects the electrical conductivity of the film and the contact between PEDOT:PSS thin film

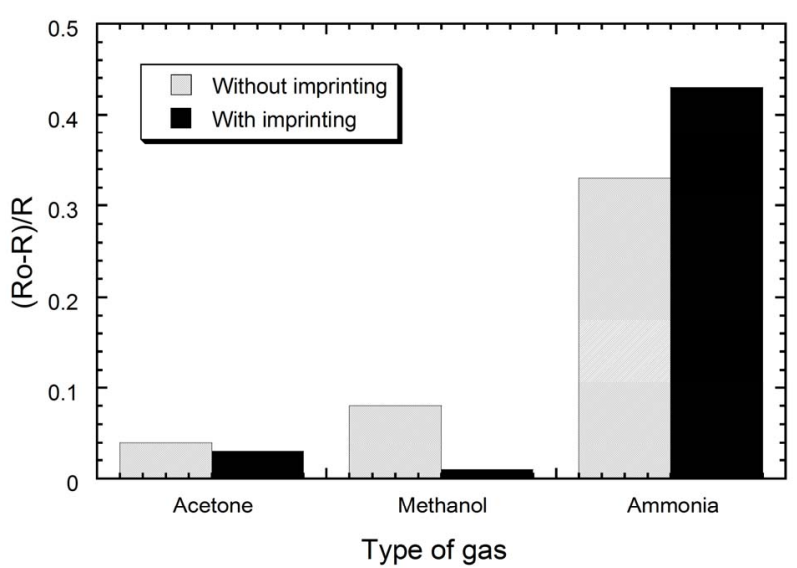

Figure 8. Selectivity of sensors to different types of gas.

and Au electrodes is found to be an ohmic characteristic. When the imprinted sensor is exposed in ammonia gas, the sensor has the short response and recovery time that is 10 seconds and 15 seconds, respectively, comparing the results of Dan et al. [9]. The sensor also has a good repeatability where the response of sensor is reversible and higher sensitivity to ammonia gas. Our fabricated sensor using the imprinting method can improves the selectivity of the sensor to ammonia gas. The results suggest that the sensor might be suitable for single sensor to the gas. Further study to fabricate the sensor with our method using another gas as imprinted material is extremely useful. The works in relation to these problems are still in progress.

\section{Acknowledgements}

This work is supported by Gadjah Mada University through Doctoral Dissertation Research Grant, No: LPPMUGM/888/BID.1/2011.

\section{REFERENCES}

[1] J. Janata and M. Josowicz, "Conducting Polymers in Electronic Chemical Sensors,” Nature Materials, Vol. 2, No. 1, 2003, pp. 19-24.

[2] J. W. Gardner, V. K. Varadan and O. O. Awadelkarim "Microsensors, MEMS and Smart Devices," John Wiley \& Sons Ltd., Chichester, 1994.

[3] G. K. Prasad, T. P. Radhakrishnan, D. S. Kumar and M. G. Krishna, "Ammonia Sensing Characteristics of Thin Film Based on Polyelectrolyte Templated Polyaniline," Sensors and Actuators B: Chemical, Vol. 106, No. 3, 2005, pp. 626-631. doi.10.1016/j.snb.2004.09.011,

[4] P. T. Moseley and A. J. Crocker, "Sensor Materials," Institute of Physics Publishing Ltd, Bristol, 1996.

[5] T. Miyata, S. Kawaguchi, M. Ishii and T. Minami, "High Sensitivity Chlorine Gas Sensors Using Cu-Phthalocyanine Thin Films," Thin Solid Films, Vol. 425, No. 1-2, 2003, pp. 255-259. doi.10.1016/S0040-6090(02)01129-X 
[6] D. Nicolas-Debarnot and F. Poncin-Epaillard, "Polyaniline as a New Sensitive Layer for Gas Sensors," Analytica Chimica Acta, Vol. 475, No. 1-2, 2003, pp. 1-15. doi.10.1016/S0003-2670(02)01229-1

[7] H. Bai and G. Shi, "Gas Sensors Based on Conducting Polymers,” Sensors, Vol. 7, No. 3, 2007, pp. 267-307. doi.10.3390/s7030267

[8] T. M. Schweizer, "Electrical Characterization and Investigation of the Piezoresistive Effect of PEDOT:PSS Thin Films," Master Thesis, Georgia Institute of Technology, 2005.

[9] Y. Dan, Y. Cao, Tom E. Mallouk, A. T. Johnson and S. Evoy, "Dielectrophoretically Assembled Polymer Nanowires for Gas Sensing," Sensors and Actuators B, Vol. 125, No. 1, 2007, pp. 55-59. doi.10.1016/j.snb.2007.01.042.

[10] H. Yan and K. H. Row, "Characteristic and Synthetic Approach of Molecularly Imprinted Polymer," International Journal of Molecular Sciences, Vol. 7, No. 5, 2006, pp. 155-178. doi.10.3390/i7050155

[11] Khairurrijal, M. Abdullah, A. Suhendi, M. M. Munir and A. Surachman, “A Simple Microcontroller-Based Current Electrometer Made from LOG112 And C8051F006 for
Measuring Current in Metal-Oxide-Semiconductor Devices,” Measurement Science and Technology, Vol. 18, No. 9, 2007, pp. 3019-3024. doi:10.1088/0957-0233/18/9/037.

[12] Khairurrijal, M. Abdullah, A. Suhendi, M. M. Munir and A. Surachman, "Low Cost and User-friendly Electronic Components Characterization System for Undergraduate Students," WSEAS Transactions on Advances in Engineering Education, Vol. 3, No. 11, 2006, pp. 971-976.

[13] H. Chen, "Modulation Effects on Organic Electronics," Ph.D. Thesis, Georgia Institute of Technology, 2005.

[14] H. J. Zheng, Y. D. Jiang, J. H. Xu and Y. J. Yang, “Gas Sensitivity of Poly (3,4-ethylene dioxythiophene) Prepared by a Modified LB Film Method,” Journal of Wuhan University of Technology-Material Science Edition, Vol. 26, No. 1, 2011, pp. 70-74.

[15] C.-Y. Lin, J.-G. Chen, C.-W. Hu, J. J. Tunney and K.-C. Ho, "Using a PEDOT:PSS Modified Electrode for Detecting Nitric Oxide Gas," Sensors and Actuators B: Chemical, Vol. 140, No. 2, 2009, pp. 402-406. doi.10.1016/j.snb.2009.04.041. 